\title{
Análisis del precio de los futbolistas de la liga premier inglesa con respecto a sus estadisticas en FIFA 20
}

\section{Price analysis of the english premier league soccer players according to their FIFA 20 statistics $^{1}$}

\author{
Gonzalo Rafael Moya Villanueva ${ }^{2}$ \\ Universidad Nacional Mayor de San Marcos \\ gmovav@unmsm.edu.pe \\ Luis Antonio Córdova Ramos ${ }^{3}$ \\ Universidad Nacional Mayor de San Marcos \\ luis.cordova8@unmsm.edu.pe
}

Recibido: 15/09/2020 - Aceptado: 6/09/2021 - Publicado: 06/12/2021

\begin{abstract}
RESUMEN
Partimos de la premisa de que el videojuego FIFA 20 mide correctamente las habilidades de los futbolistas profesionales, y con estas mediciones construimos un modelo econométrico para identificar qué jugadores estarían siendo sobrevaluados y cuáles sub-estimados, comparando sus cotizaciones en la página web TransferMarkt contra los valores que les asigna nuestro modelo basado en sus destrezas. Se tomó una muestra de los 14 jugadores con más minutos de juego en cada uno de los 20 clubes que participaron en la Premier League 2019-20 hasta la suspensión del campeonato por la pandemia (sin incluir a los arqueros). Como resultado obtuvimos que, si bien la ubicación de los clubes en la tabla de posiciones tiene una correlación más positiva con las cotizaciones de sus planteles en TransferMarkt que con sus habilidades medidas por FIFA20, la observación de estas discrepancias entre precio y valor sí generaría una ventana de oportunidad
\end{abstract}

(C) Los autores. Este artículo es publicado por Pensamiento Crítico de la Facultad de Ciencias Económicas, Universidad Nacional Mayor de San Marcos. Este es un artículo de acceso abierto, distribuido bajo los términos de la licencia Creative Commons Atribucion - No Comercia_Compartir Igual 4.0 Internacional. (http://creativecommons.org/licenses/by-nc-sa/4.0/) que permite el uso no comercial, distribución y reproducción en cualquier medio, siempre que la obra original sea debidamente citada. 
para armar equipos de manera inteligente y conseguir así la eficiencia en los gastos de contratación.

Palabras clave: Regresión Múltiple; Liga Premier Inglesa; TransferMarkt; FIFA 20. Clasificación JEL: C10 (Métodos y Metodología Econométrica y Estadística: General); Z20 (Economía del Deporte).

Reconocimiento: Este trabajo está dedicado con cariño a nuestras familias, quienes soportan y apoyan nuestra pasión tanto por el fútbol como por la Economía. Agradecemos también a nuestros colegas en la UNMSM y a nuestros árbitros anónimos por sus valiosas sugerencias para mejorar nuestro ensayo. Gonzalo Moya pertenece al grupo de investigación "Dynamic”, liderado por el Decano Richard Roca.

\section{ABSTRACT}

On the basis of the premise that the videogame FIFA 20 measures correctly the abilities of professional soccer players, we built an econometric model with these measurements to identify which players would be over-priced and which ones under-estimated, comparing their quotings at the TransferMarkt website against the values assigned by our model based on their skills. We took a sample of the 14 players with more minutes on field that participated on the 2019-20 Premier League until the stoppage due to the pandemic (not including goalkeepers). As a result, we obtained that, although the clubs' placement on the standings table had a more positive correlation with their staff quotings at TransferMarkt than with their abilities measured by FIFA 20, looking for discrepancies between price and value does indeed generate a window opportunity to build teams in a smart way, and thus achieve efficiency on hiring expenditures.

Keywords: Multiple Regression; English Premier League; TransferMarkt; FIFA 20.

JEL Classification: C10 (Econometric and Statistical Methods and Methodology: General), Z20 (Sports Economics: General).

Acknowledgements: This work is dedicated with love to our families, who stand and support our passion both for Soccer and for Economics. We also thank our colleagues at UNMSM and the anonymous referees for their valuable suggestions towards improving our paper. Gonzalo Moya belongs to the research group "Dynamic", led by Dean Richard Roca. 


\section{Introducción}

Como diría Raúl Rosales, Director de la carrera de Administración y Negocios del Deporte en la UPC, "[l]as investigaciones académicas que buscan encontrar la relación entre logro deportivo y presupuesto asignado han concluido que a mayor presupuesto, mayor es la probabilidad de éxito deportivo" (Rosales). El Bayern Múnich en la Bundesliga de Alemania, la Juventus de Turín en la Serie A de Italia, y más recientemente, el París Saint-Germain en la Ligue 1 de Francia, son ejemplos de clubes que han hegemonizado las ligas de fútbol profesional de sus respectivos países a partir de su poderío económico.

La presente investigación académica no pretende pues, redundar en una conclusión que para empezar nunca desafió al sentido común. Después de todo, el dinero no solo te permite comprar a los mejores jugadores y director técnico, sino también al resto del personal de apoyo y demás material logístico detrás de los protagonistas, para que ellos simplemente se dediquen a hacer lo que mejor saben. El presente ensayo lo que pretende más bien es hacer una reflexión sobre las escasas excepciones a esta regla tan obvia, aquellas raras anomalías cuando el poder económico de los clubes no concuerda con su rendimiento en los torneos en los que participan.

A veces suceden excepciones felices, en los que un equipo de relativamente bajo presupuesto (una "Cenicienta") sorprende gratamente, obteniendo resultados mejores de los esperados y peleando los primeros puestos de la clasificación (tal es el caso reciente del Atalanta en Italia), o saliendo campeón inclusive (como le pasó en el 2016 al Leicester en Inglaterra). Entonces se vuelven noticia y motivan columnas por cómo lograron reavivar, aunque brevemente, la visión romántica de que el Deporte puede ser menos mecánico que las demás industrias, y más impredecible que el resto de ciencias.

Sobre el primero, el periodista Raúl Castillo escribió que "En una época en la que parece que la institución que cuenta con más presupuesto se lleva los tres puntos, el Atalanta de Bérgamo le ha devuelto la ilusión a miles de hinchas en todas las partes del mundo. La billetera, con ello, no necesariamente mata una idea de juego y todo se vuelve posible en un campo entre 22 jugadores durante 90 minutos de fútbol" (Castillo). Sobre el segundo, el Prof. Rosales en la misma columna proseguía: "[E]l 
Leicester, un equipo de menor presupuesto [con un grupo de jugadores que no estaban dentro de la lista de los más cotizados del mercado, este club situado entre Londres y Mánchester] está haciendo una campaña inolvidable que pone en tela de juicio que los planteles caros sean los que estén destinados a campeonar" (Rosales).

A veces, sin embargo, ocurren otro tipo de anomalías, aciagas, en las que un equipo de presupuesto relativamente alto (un "grande") desconcierta a todos al obtener resultados peores a los que se esperaban tanto por simpatizantes como por contrarios, y terminan peleando por salvar la categoría, a veces incluso sin éxito para esto último. El ejemplo más cercano y reciente de ello, qué duda cabe, es el de Alianza Lima ${ }^{4}$, quizá el equipo más "popular" del Perú (el de mayor tradición y cantidad de aficionados).

Esta noticia también ha motivado muchas columnas, claro que escritas con enojo y desahogo en vez de algarabía: El periodista Christian Cruz, por ejemplo, al día siguiente de concretado el descenso, y aún con el dolor de la derrota por 2-0 frente a Sport Huancayo en la fecha final, anotó: "Cuatro puntos de los últimos 36 en juego. Y un solo gol en los últimos cuatro partidos, en los que solo necesitaba ganar uno. Ni de eso fue capaz" (Cruz[1]). Luego, casi un mes después, añadiría: "En los 27 partidos que disputó, los íntimos marcaron solo 25 goles. [...] Alianza recibió un total de 36 goles (solo seis veces pudo mantener su arco en cero) y [...] solo logró cinco victorias" (Cruz [2]).

Y aunque las estadísticas que presenta son contundentes, éstas simplemente describen la magnitud de la tragedia, mas no logran explicarla, como sí lo intenta hacer su colega Eduardo Combe, quien marca como punto de quiebre que desbalanceó el plantel cuando el uruguayo "Adrián Balboa dejó el equipo por el término de su contrato, acompañado por sus compatriotas Luis Aguiar y Federico Rodríguez. Nunca quedó en claro por qué estos dos últimos decidieron rescindir. Aldair Fuentes había sido fichado en la Segunda División de España. [...] Cristian Zúñiga, la contratación extranjera de más bajo rendimiento en años, también decidió dejar tienda blanquiazul." (Combe).

El editor Pedro Canelo retrocede aún más en el tiempo, hasta antes de la interrupción por la pandemia del coronavirus, en su afán por encontrar los momentos críticos que dieron origen a la actual crisis institucional. Las 
contrataciones de Jean Deza y Carlos Ascues resultaron ser nefastas para el club, no solo por su bajo rendimiento en el campo de juego, sino y sobre todo, porque sus constantes indisciplinas contagiaron de desdén (indiferencia, despego) y dejadez (pereza, negligencia) al resto del plantel. Tardaron demasiado en separar al primero, y el segundo terminó siendo el jugador símbolo del descenso, pero ambos habían sido pedidos expresos del entonces entrenador Pablo Bengoechea, quien ya los había convocado cuando fue director técnico de la selección peruana. En cambio, dice el editor, "[los del Fondo Blanquiazul] no pueden evadir responsabilidades en otras dos llegadas muy criticadas como las del colombiano Cristian Zúñiga y, sobre todo, la de Beto da Silva. Ese refuerzo sí merece ser explicado por el fondo. Al delantero le hicieron un contrato por tres años cuando su actividad futbolística carecía de regularidad por más de tres años" (Canelo).

Ambos tocan aspectos claves que son consistentes con la hipótesis principal de este ensayo, a decir: Que los clubes que a pesar de su alto presupuesto no logran los resultados esperados son aquellos que estuvieron lejos de alcanzar la eficiencia en su gasto, trayendo jugadores sobrevaluados (cuyo talento o destreza es menor al que corresponde según su precio), y dejando ir a los que están sub-estimados (cuyas habilidades exceden a su cotización). Por contraposición, los clubes que con su bajo presupuesto logran superar ampliamente las expectativas son aquellos que sí alcanzaron la eficiencia en el gasto, trayendo jugadores sub-estimados y dejando ir a los que están sobre-valuados.

La aplicación de este principio elemental de las Finanzas a los deportistas profesionales en general es la base de lo que se conoce como "sabermetrics" ("métricas-sable"), sobre la cual existe abundante literatura (tanto académica como de no-ficción), y hasta una película (protagonizada por Brad Pitt, nada menos). El aporte de este ensayo pasa por el hecho de que, si todos los clubes fuesen "racionales" (en el sentido económico del término), buscarán su propio beneficio optimizando individualmente la eficiencia de su gasto, vendiendo aquellos jugadores que consideren sobre-valuados y comprando a los crean que están sub-estimados.

Lo que es más importante, si todos los clubes realizaran este arbitraje utilizando los mismos criterios para cotizar a los jugadores, los clubes coincidirían sobre quiénes son los jugadores que están sobre-valuados y 
cuáles están sub-estimados. Se aplicaría así la Hipótesis de los Mercados Eficientes también en los deportes, donde todos los jugadores, como por una mano invisible, tenderían a valorizarse a la par de sus habilidades, es decir, sus cotizaciones se alinearían de acuerdo a sus niveles de talento o destreza, como se muestra en la figura debajo. De este modo, los clubes que contraten demasiado bien (como el Atalanta o Leicester) o demasiado mal (como Alianza Lima), terminan siendo la excepción y no la regla (ver Figura $\left.N^{\circ} 1\right)$.

\section{Figura $\mathbf{N}^{\circ} \mathbf{1}$}

Cotizaciones en TransferMarkt ("y", eje vertical) en función del valor del jugador según sus estadísticas en FIFA 20 ( " $y^{\wedge "}$ ", eje horizontal)

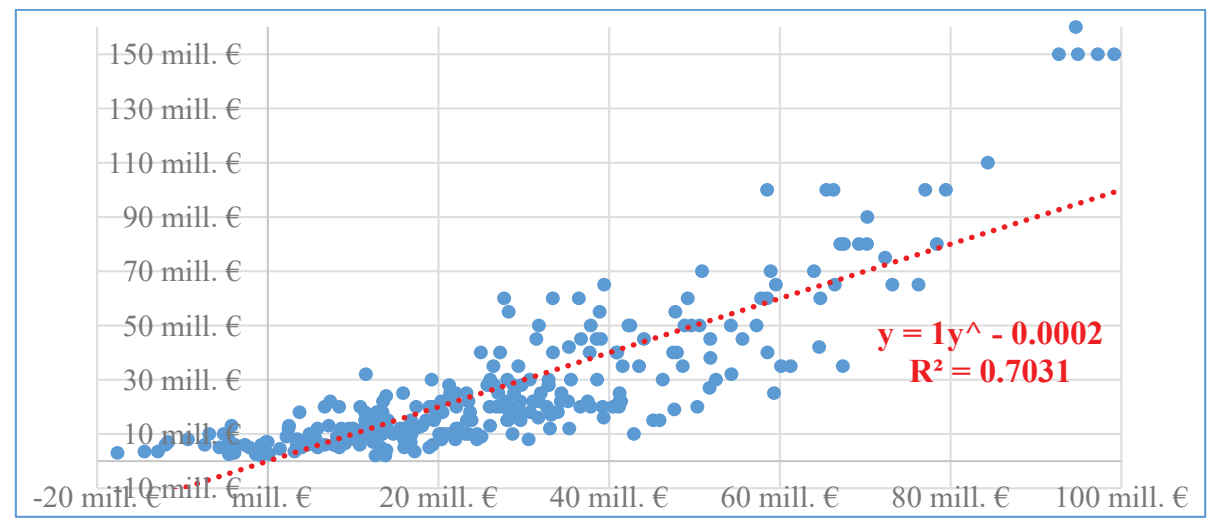

Fuente: Elaboración propia.

La estructura del ensayo es la siguiente: La sección 2 contiene el marco teórico, pues discute la metodología (y revisa la literatura disponible) sobre "métricas-sable" para una valoración de los jugadores de béisbol y su aplicación a otros deportes profesionales tales como el fútbol. La sección 3 presenta a la Liga Premier inglesa. La sección 4 introduce a la página web TransferMarkt, la cual es de donde obtuvimos la data para nuestra variable explicada: las cotizaciones de los jugadores de fútbol profesional, y presenta el videojuego de FIFA 20, el cual es de donde obtuvimos la data para nuestras variables explicativas: mediciones numéricas de las habilidades para el fútbol de los jugadores profesionales, con los que estimar el precio justo del jugador, con lo cual la variabilidad que no es predicha por el modelo constituirá su sobre-valuación o sub-estimación. 
La sección 5 presenta los resultados de tal modelo econométrico, una ecuación de regresión múltiple con las cotizaciones de los jugadores de fútbol profesional en función de sus habilidades para el fútbol. La sección 6 analiza este modelo al declarar que, si las habilidades fueron correctamente medidas por FIFA 20, entonces los precios que muestra TransferMarkt de hecho te indican qué jugadores están sobre-valuados y cuáles están sub-estimados. La sección 7 concluye este ensayo al continuar con las interpretaciones de los resultados del modelo y señalar la dirección hacia investigaciones posteriores.

\section{Marco Teórico}

Pelota de dinero: El arte de ganar un juego injusto.

En el 2003, Michael Lewis publicó su libro "Moneyball: The Art of Winning an Unfair Game", el cual cuenta la historia de Billy Beane, un gerente deportivo del Oakland Athletics (un club de béisbol de los Estados Unidos) quien, con la ayuda de su asistente, el economista de Harvard Paul DePodesta, ensambló un nuevo equipo ganador para la temporada 2002, luego de que el anterior fuese desmantelado por los clubes financieramente poderosos de la Liga Mayor de Béisbol (“MLB” por siglas en inglés). Para lograr este objetivo dado su reducido presupuesto, buscaron a los jugadores más sub-estimados de la MLB de acuerdo a un modelo matemático desarrollado por ellos mismos, el cual incluía medidas previamente no consideradas, tales como el número de veces que los bateadores llegaron a primera base, sin importar cómo (Lewis, 2004).

Beane y DePodesta fueron con su análisis estadístico contra el método tradicional de los reclutadores (cazatalentos), quienes se enfocaban más en aspectos cualitativos que cuantitativos sobre qué jugadores contratar: su valoración de los beisbolistas podía basarse en criterios bastante subjetivos, si no en meras corazonadas. A pesar de que al comienzo parecía que el proyecto de Beane y DePodesta sería un completo fracaso (pues desechaba casi por completo aspectos cualitativos que también eran importantes), finalmente resultó ser bastante exitoso. Desde entonces su metodología de "métricas-sable" ("sabermetrics") ha ganado una amplia aceptación y respeto dentro de la gestión deportiva, al punto de que un "antes y después" en las técnicas de valoración de los jugadores de 
béisbol profesional es claramente identificable a partir del año en el que Lewis publicó su libro. (Chang \& Zenilman, 2013) (ver Figura $\mathrm{N}^{\circ} 2$ )

\section{Figura $\mathrm{N}^{\circ} 2$}

Moneyball: portada del libro (izquierda) y de la película (derecha).

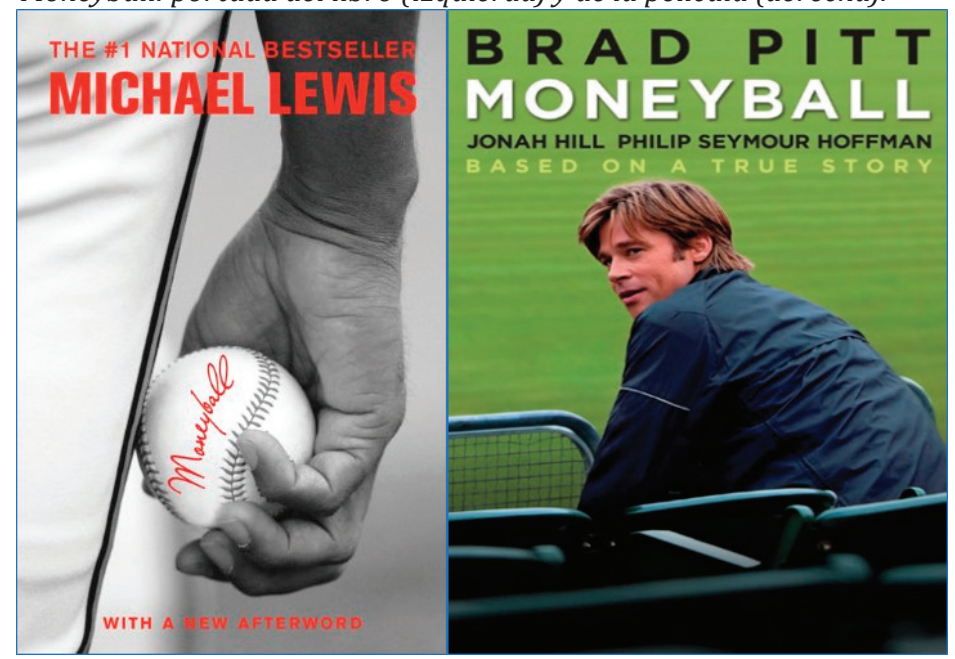

Fuente: Amazon; Sony Pictures

Más aún, en el 2004 la empresa cinematográfica Columbia Pictures adquirió los derechos de autor del libro y en el 2011, luego de siete años en desarrollo, lanzó una película basada en este, titulada "Moneyball: Rompiendo las reglas" en España, y "Moneyball: El juego de la fortuna” en Latinoamérica. La película fue dirigida por Bennet Miller con guion adaptado de Steven Zaillian y Aaron Sorkin, protagonizada por Brad Pitt como Billy Beane, Jonah Hill como "Peter Brand" (/Paul DePodesta), y Phillip Seymour Hoffman como el entrenador Art Howe. Obtuvo 6 nominaciones al Óscar, incluyendo Mejor Película y Mejor Guión Adaptado (IMDb, FilmAffinity).

Con el paso del tiempo, se han aplicado variantes de las métricas-sable a otros deportes profesionales colectivos, tales como el fútbol, al punto que el mismo Billy Beane se declaró admirador del ex gerente deportivo del Arsenal FC, Arsene Wegner (Bascombe). Más recientemente, se destaca el trabajo en la misma línea por parte del co-dueño del Liverpool, John W. Henry y su director técnico, Jurgen Klöp (Maupomé). Fuera del Inglaterra, sobresalen los desempeños de los cazatalentos del Borussia Dortmund de 
Alemania, Michael Zorc, y del Sevilla de España, Ramón Rodríguez “Monchi” (Barraza [2]), solo por mencionar a algunos.

Dentro de la literatura académica, cabe destacar el trabajo de Benjamin Baumer y Andrew Zimbalist en "The Sabermetric Revolution", quienes critican la película de Moneyball por exagerar la subjetividad de los métodos tradicionales al punto del ridículo, tales como revisar la belleza de la novia del jugador para estimar su nivel de auto-confianza en el campo. Lo que sí reconocen es que si bien antes ya se consideraba ciertos aspectos cuantitativos, se ponderaba demasiado el promedio de bateo del jugador, y que el aporte de Bean y DePodesta de empezar a incluir la cantidad de veces en que el bateador llegaba a primera base (sin importar cómo) sí fue algo insólito hasta entonces, y revolucionario a partir de allí (Baumer \& Zimbalist, 2014).

Los autores dan en otro punto clave para nuestro ensayo: Al final de la Introducción dijimos que, si todos los clubes utilizaban los mismos criterios para evaluar a los jugadores, estarían de acuerdo en considerar sobre-valuados o sub-estimados a quienes lo estuviesen y esto a su vez iba a llevar a que todos los jugadores estén justamente cotizados según sus habilidades. Como todos los clubes contrataban de manera eficiente pero dentro de sus propios presupuestos, no dejarían lugar a sorpresas en la tabla de posiciones. Por ende, para que un club pueda sorprender al resto, necesariamente debe salirse del esquema y encontrar la forma de cotizar a los jugadores de manera distinta, con el riesgo de hacerlo excepcionalmente bien (como los Oakland Athletics de Bean y DePodesta) o excepcionalmente mal (de nuevo, como la Alianza Lima del Fondo Blanquiazul).

Nuestro ensayo propone una manera distinta de valorizar a los futbolistas, que nos permita encontrar qué jugadores no estarían realmente cotizados a la par de sus talentos y destrezas, y abrir así una ventana de posibilidad para que un club de presupuesto relativamente modesto para la liga en que compite pueda dar una sorpresa grata en cuanto a logros deportivos se refiere.

\section{La Liga Premier Inglesa 2019-2020.}

Ciertamente, pudimos tomar cualquiera de las principales ligas de fútbol profesional en Europa, pero nuestra elección por la primera división de 
Inglaterra es explicada mejor por el columnista deportivo Jorge Barraza: "Desde hace muchos años el inglés es el medio más atractivo del mundo por historia, encanto, escenarios, por organización, pulcritud y puesta en escena, por ese decorado subyugante que rodea a un estilo a veces básico en el terreno táctico, pero vertical, entregado, franco. Y últimamente también es el fútbol más lucido y ganador." (Barraza [1])

Nadie es indiferente al encanto de la Premier League, a su valor estético y su limpieza moral. De acuerdo al autor, la principal razón por la cual resulta tan edificante disfrutar de su generosidad futbolística tendría una explicación económica: "[L]os más poderosos pueden fichar mejor y tienen mayores posibilidades. Sin embargo, es la única liga importante que reparte sus ingresos por derechos de televisación en partes iguales entre los 20 competidores de Primera División. Esto, con el objeto de nivelar al máximo las posibilidades de todos[,] permite que todos puedan reforzarse y dar batalla." (Barraza [1])

Su artículo se escribió al día siguiente de la final de la FA Cup 20192020, que se disputó recién el 1 de agosto, jugado sin público y pospuesto debido a la pandemia del coronavirus. Como dato curioso, ambos finalistas de esa edición, el Chelsea y el Arsenal, fueron equipos de la capital Londres que llegaron a esa instancia luego de eliminar en semi-finales a los dos de Manchester, el United y el City, respectivamente. Con doblete de Pierre-Emerick Aubameyang, el Arsenal ganó 2 a 1 y conquistó así su título \#14 de este torneo, en el que si bien participan clubes de las diez divisiones del fútbol inglés, para cuartos de final ya solo llegan los de primera, como se aprecia en la Figura $N^{\circ} 3$.

La extensa columna de Jorge Barraza concluye así: "La búsqueda de la excelencia, tan típicamente inglesa, está omnipresente en cada detalle organizativo, en el producto televisivo, que sale al mundo empacado por la propia liga. Pero, por encima de la estética y el orden, presidiéndolo todo, se evidencia una alta estatura moral. Que excede lo deportivo y nos atañe como sociedad, a cómo encaramos la vida diaria en todas las actividades." (Barraza [1])

De hecho, la última versión del presente ensayo, que subsana las observaciones hechas por nuestros pares académicos en sus informes de revisión, se finalizó el 26 de diciembre del 2020, que coincide con el “Boxing 
Day" ("Día de las Cajas"), una tradición del fútbol inglés que se realiza oficialmente desde 1888, "como un obsequio de la Federación Inglesa para los aficionados al deporte, con ofertas de tickets y otros descuentos. Aquella campaña se estaba inaugurando la pionera Football League" (Barrio de Mendoza).

\section{Figura $\mathbf{N}^{\circ} 3$}

El camino hacia la FA Cup 2019-2020.

\begin{tabular}{|c|c|c|c|}
\hline Octavos de final & Cuartos de final & Semifinales & Final \\
\hline \multicolumn{4}{|l|}{ Tottenham Hotspur (1) } \\
\hline Norwich City (1)p & Norwich City (1) & & \\
\hline \multirow{2}{*}{$\begin{array}{c}\text { Derby County (0) } \\
\text { Manchester United (3) }\end{array}$} & Manchester United (2) & Manchester United (1) & \\
\hline & & & \\
\hline \multirow{2}{*}{$\begin{array}{c}\text { Leicester City (1) } \\
\text { Birmingham City (0) }\end{array}$} & & & Chelsea (1) \\
\hline & Leicester City (0) & Chelsea (3) & \\
\hline Chelsea (2) & Chelsea (1) & & \\
\hline \multicolumn{4}{|l|}{ Liverpool (0) } \\
\hline \multicolumn{4}{|l|}{ West Bromwich (2) } \\
\hline \multirow{2}{*}{ Sheffield Wednesday (0) } & Newcaslte United (0) & & \\
\hline & Manchester City (1) & Manchester City (0) & \\
\hline Manchester City (1) & & & Arsenal (2) \\
\hline \multirow{2}{*}{$\begin{array}{c}\text { Reading (1) } \\
\text { Sheffield United (2) }\end{array}$} & & & \\
\hline & Sheffield United (1) & Arsenal (2) & \\
\hline \multirow{2}{*}{$\begin{array}{l}\text { Portsmouth (0) } \\
\text { Arsenal (2) }\end{array}$} & Arsenal (2) & & \\
\hline & & & \\
\hline
\end{tabular}

Fuente: Wikipedia. FA Cup 2019-20. Elaboración propia

La Tabla $\mathrm{N}^{\circ} 1$ muestra la tabla de posiciones al término de la edición 2019-2020 de la Premier League (llamada así desde 1992). La nota "a" en Arsenal es porque clasificó a la Europa League, al igual que los demás equipos en naranja, pero por haber ganado la FA Cup, como se mencionó en la página anterior. En azul están los que clasificaron a la Champions League y en rojo los que descendieron de categoría. Puede verse la disparidad en el ordenamiento de las posiciones entre ambos torneos: El Liverpool, por ejemplo, salió campeón en la Premier League (en el que juegan todos contra todos en 38 partidos de ida y vuelta solo los 20 equipos de primera), mientras que quedó eliminado en octavos de final de la FA Cup (donde juegan a un único partido en llaves de eliminación directa los equipos de las diez categorías). 


\section{Tabla $\mathbf{N}^{\circ} \mathbf{1}$}

Tabla de posiciones al término de la Premier League 2019-2020.

\begin{tabular}{|c|c|c|c|c|c|c|c|c|c|c|}
\hline Pos. & Equipo & Pts. & PJ & G & $\mathbf{E}$ & $\mathbf{P}$ & GF & GC & Dif. & Clasificación 2020-2021 \\
\hline 1 & Liverpool (C) & 99 & 38 & 32 & 3 & 3 & 85 & 33 & +52 & \\
\hline 2 & Manchester City & 81 & 38 & 26 & 3 & 9 & 102 & 35 & +67 & Fase de grupos de la Liga de \\
\hline 3 & Manchester United & 66 & 38 & 18 & 12 & 8 & 66 & 36 & +30 & Campeones \\
\hline 4 & Chelsea & 66 & 38 & 20 & 6 & 12 & 69 & 54 & +15 & \\
\hline 5 & Leicester City & 62 & 38 & 18 & 8 & 12 & 67 & 41 & +26 & Fase de grupos de la Liga Europa \\
\hline 6 & Tottenham Hotspur & 59 & 38 & 16 & 11 & 11 & 61 & 47 & +14 & Segunda ronda de la Liga Europa \\
\hline 7 & Wolverhampton Wanderers & 59 & 38 & 15 & 14 & 9 & 51 & 40 & +11 & \\
\hline 8 & Arsenal $^{\mathrm{a}}$ & 56 & 38 & 14 & 14 & 10 & 56 & 48 & +8 & Fase de grupos de la Liga Europa \\
\hline 9 & Sheffield United & 54 & 38 & 14 & 12 & 12 & 39 & 39 & 0 & \\
\hline 10 & Burnley & 54 & 38 & 15 & 9 & 14 & 43 & 50 & -7 & \\
\hline 11 & Southampton & 52 & 38 & 15 & 7 & 16 & 51 & 60 & -9 & \\
\hline 12 & Everton & 49 & 38 & 13 & 10 & 15 & 44 & 56 & -12 & \\
\hline 13 & Newcastle United & 44 & 38 & 11 & 11 & 16 & 38 & 58 & -20 & \\
\hline 14 & Crystal Palace & 43 & 38 & 11 & 10 & 17 & 31 & 50 & -19 & \\
\hline 15 & Brighton \& Hove Albion & 41 & 38 & 9 & 14 & 15 & 39 & 54 & -15 & \\
\hline 16 & West Ham United & 39 & 38 & 10 & 9 & 19 & 49 & 62 & -13 & \\
\hline 17 & Aston Villa & 35 & 38 & 9 & 8 & 21 & 41 & 67 & -26 & \\
\hline 18 & Bournemouth (R) & 34 & 38 & 9 & 7 & 22 & 40 & 65 & -25 & \\
\hline 19 & Watford (R) & 34 & 38 & 8 & 10 & 20 & 36 & 64 & -28 & Descenso de Categoría \\
\hline 20 & Norwich City (R) & 21 & 38 & 5 & 6 & 27 & 26 & 75 & -49 & \\
\hline
\end{tabular}

(Fuente: Wikipedia. Premier League 2019-20)

\section{TransferMarkt y FIFA 20}

\section{La página web TransferMarkt}

Transfermarkt GmbH \& Co. KG es una empresa ubicada en Hamburgo, Alemania, cuya página web "TransferMarkt" brinda información actualizada de las competencias de fútbol más importantes del mundo. Aparte de noticias sobre fichajes concretados y rumores sobre posibles traspasos, la página se destaca por brindar las cotizaciones de los jugadores profesionales. 
Es decir, el precio que un club tendría que pagar para comprar a un jugador o el dinero que el club que posee a ese jugador podría obtener si lo vendiese.

Por ejemplo, en cuanto a los jugadores que más subieron de valor al término de la temporada 2019-2020, está el lateral izquierdo canadiense del Bayern Munich, Alphonso Davies, que de costar €25 millones pasó a valer €60 millones (+€35 millones ó 140\%), luego de una destacada participación en la eliminación del Barcelona por los cuartos de final de la Champions League. Otro caso parecido es el del centro delantero noruego del Borussia Dormund, Erling Haaland, que de costar €45 millones pasó a valer €72 millones (+€27 millones ó 60\%), tras una notable actuación en la llave de octavos final de la Champions League, a pesar de que su equipo terminase siendo eliminado por el Paris Saint-Germain.

Por otro lado, en cuanto a los jugadores que más bajaron de valor al término de la temporada 2019-2020, está el extremo izquierdo belga del Real Madrid, Eden Hazard, que de costar €120 millones pasó a valer €80 millones (-€40 millones ó -33\%), luego de una intrascendente participación en la llave de octavos de final de la Champions League, donde su equipo quedó eliminado frente al Manchester City. Otro caso similar es el del extremo izquierdo brasileño del Paris Saint-Germain, Neymar Jr., que de costar €160 millones pasó a valer €128 millones (-€32 millones ó -20\%), tras una discreta actuación en la final de la Champions League, donde su equipo cayó derrotado ante el Bayern Múnich.

En general, sin embargo, la pandemia ha traído una recesión económica a nivel mundial del cual el fútbol no ha sido ajeno. Puede verse en la figura debajo que la mayoría de actualizaciones en las cotizaciones de los jugadores ha sido hacia la baja, lo que refleja una contracción en la curva de demanda futbolistas, debido a una política de austeridad por parte de los clubes, quienes esperan una menor recaudación por concepto de taquilla (ventas de boletos de entrada) y merchandising (camisetas y demás artículos con la marca del club). Incluso el mismo presidente de la FIFA, Gianni Infantino, instó a fines de marzo al gremio de clubes (ECA) y de jugadores (FIFPro) a negociar una reducción de sueldos, esto con el fin de evitar una posible quiebra de los primeros o el despido masivo de los segundos (Félix) (ver Figura $N^{\circ} 4$ ). 
Figura $\mathrm{N}^{\circ} \mathbf{4}$

La página web TransferMarkt

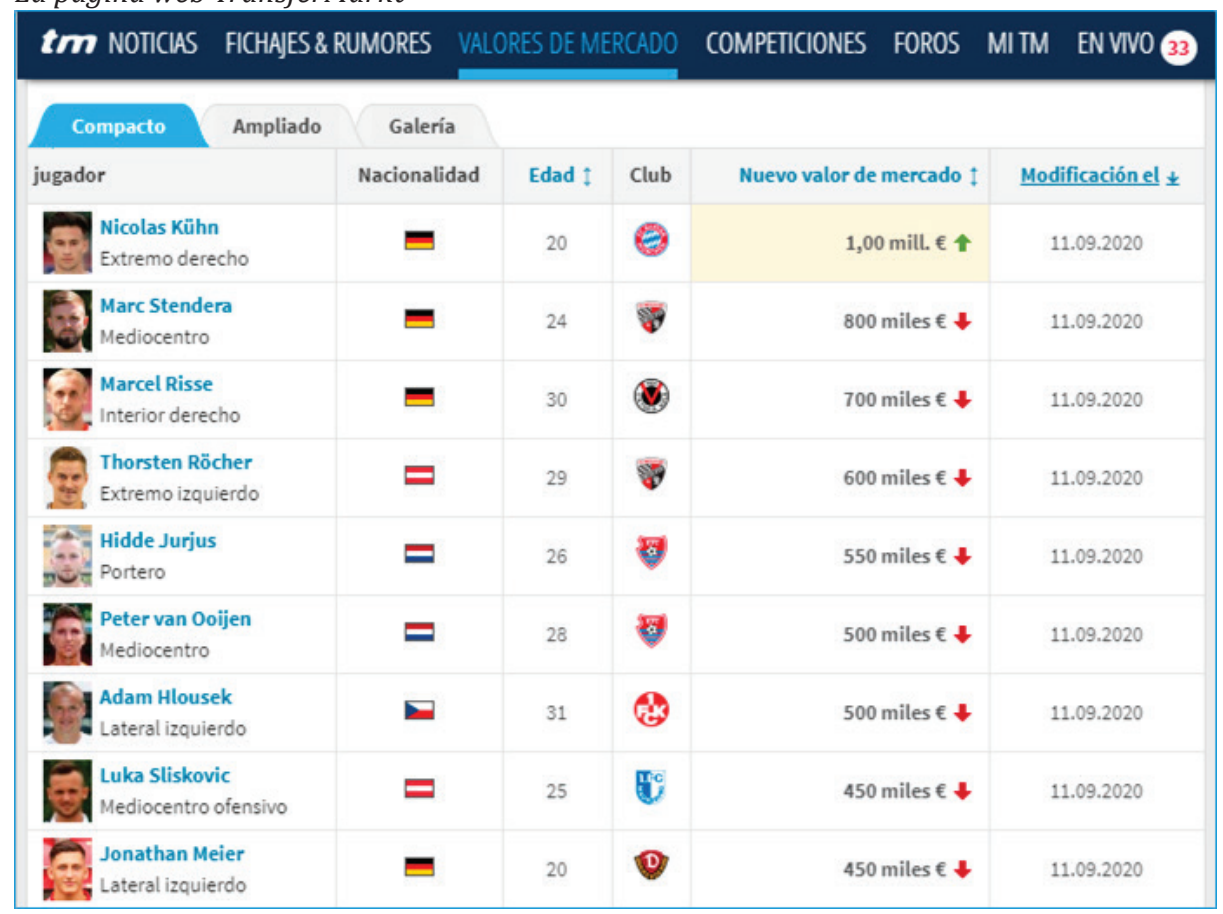

Fuente: TransferMarkt, 11/09/20

\section{El videojuego FIFA 20}

FIFA 20 es un videojuego desarrollado por la empresa Electronic Arts como parte de su serie EA Sports. El juego se llama como la Federación Internacional de Fútbol Asociado porque cuenta con su permiso para utilizar los nombres de los clubes y sus jugadores, retratando a los futbolistas estrella con un parecido creciente con la salida de nuevas consolas para videojuegos que aprovechan las mejoras tecnológicas.

Todos los años, desde el último cuatrimestre anterior (como con los nuevos modelos de automóviles), sale una nueva versión de este videojuego. Hasta FIFA 19 salió para las consolas Play Station 3 de la firma Sony y Xbox 360 de Microsoft, pero a partir de este año, FIFA 20 ha salido solo para la nueva generación de consolas: Play Station 4, Xbox One, Windows 
PC e incluso para Nintendo Switch (aunque en un Legacy Edition, que es una versión limitada del producto).

A manera de anécdota, existe una especie de "maldición" con respecto al jugador que aparece en la carátula del estuche del videojuego, que consiste en que este sufrirá una lesión de gravedad durante dicha temporada. Esta imprecación data desde sus primeras versiones, con Erik Thorstvedt en FIFA 95, Ronald de Boer en FIFA 96, Fernando Morientes en FIFA 99 y con el Pep Guardiola en FIFA 2000 (López), y ha recobrado fuerza a raíz de nuevos casos, como el de Kaká en FIFA 11, Marco Reus en FIFA 17 y Eden Hazard en FIFA 20 (El Universo).

Quizá para desafiar a la supuesta maldición (o para acabar con ella de una buena vez) es que EA Sports utilizó hasta tres jugadores distintos para cada una de las ediciones de FIFA 20, con Virgil Van Dijk y Zinedine Zidane para las otras dos (siendo el último un futbolista retirado y ahora director técnico). Para FIFA 21 volvieron a utilizar a un solo jugador, Kylian Mbappé, aunque "en un montaje múltiple que cambia por completo los moldes establecidos desde que conocemos el juego" (Pérez), como se puede apreciar en las figuras 5a y 5b. De izquierda a derecha, la Standard, Champions y Ultimate Edition.

\section{Figura $\mathbf{N}^{\circ} \mathbf{5 a}$}

Portadas de FIFA 20 para Play Station 4.

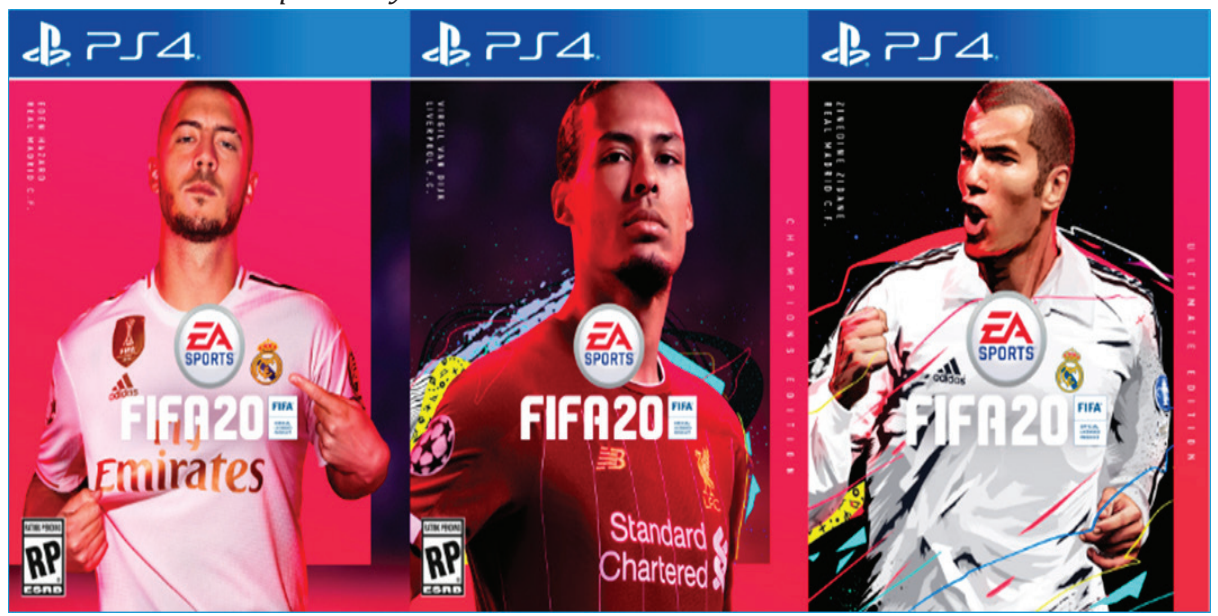


Figura $\mathbf{N}^{\circ} \mathbf{5 b}$

Portadas de FIFA 21 para Play Station 4.
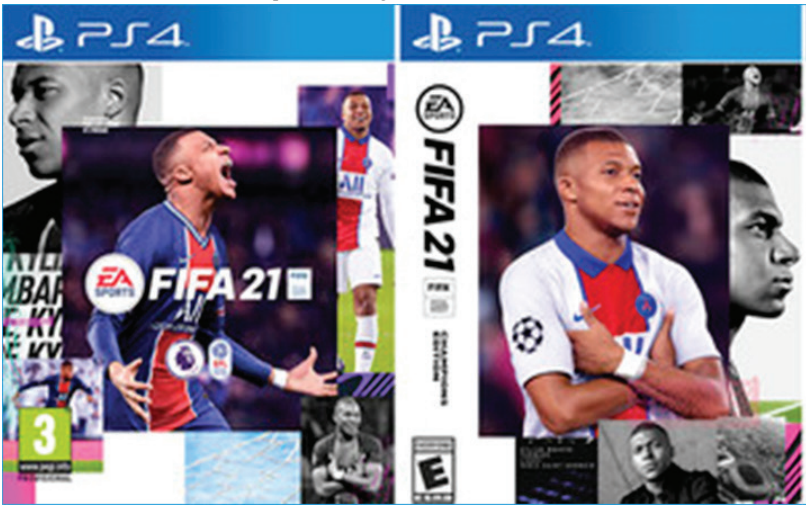

$\mathrm{CB} P \mathrm{P} 4$

CFIFA 21:

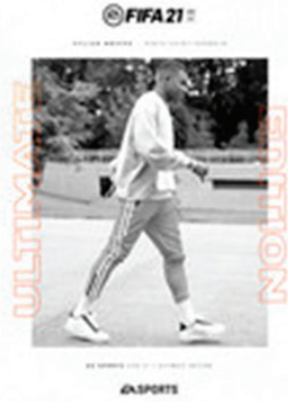

Fuentes: (a) Todo Tech 2.0, (b) VidaExtra.

Este videojuego no solo retrata a los jugadores con un parecido físico cada vez mayor, sino que establece estadísticas para ellos que afectan la "jugabilidad" del personaje. Es decir, asigna valores numéricos a un conjunto de destrezas para cada jugador, que repercuten por ejemplo, en cuán alto salta a cabecear, cuán rápido corre en los piques, o cuán fuerte patea el balón, de modo que sus habilidades en el videojuego se asemejen a las que posee en la vida real.

Dichas estadísticas se actualizan periódicamente para ajustarse a los cambios de rendimiento que los mismos jugadores vayan teniendo a lo largo de la temporada, del mismo modo en que lo hacen sus cotizaciones en TransferMarkt. Es a partir de esta observación que hemos elaborado un modelo econométrico, cuya ecuación de regresión lineal múltiple se describe en la siguiente sección.

\section{Metodología}

Para elaborar nuestro modelo econométrico, hemos puesto a la cotización de los jugadores que se publica en la página web TransferMarkt como la variable dependiente (en millones de euros), y a todas las estadísticas de destrezas en FIFA 20 como las variables independientes. Ambas datas fueron extraídas al momento del paro que hubo por la pandemia del coronavirus. La idea es que mediante esta ecuación de regresión 
múltiple modelemos el precio de los futbolistas en la vida real en función de las habilidades de sus mismos personajes en el videojuego, y atribuir la dirección del error, que es la variabilidad no explicada o predicha por el modelo, a que ese jugador está sobre-valuado o sub-estimado.

Para seleccionar la muestra hemos tomado a los 14 jugadores de campo más utilizados de cada equipo, los que más minutos de juego estaban teniendo durante la temporada hasta el momento en que se suspendió el campeonato por motivo de la pandemia. No se incluyeron a los arqueros debido a que ellos tienen diferentes indicadores de habilidad en FIFA20. Siendo 20 clubes los que conforman la Premier League, eso nos dio el total del tamaño de la muestra, de 280 jugadores de campo.

Aquí debemos hacer una acotación: FIFA 20 asignaba hasta tres posiciones de juego para un mismo futbolista, mientras que TransferMarkt les asigna solo una, de modo que la variable explicada (la cotización de los jugadores en mill. de €) no fue lo único que se tomó de esta página web, sino también la data sobre la posición de juego de cada futbolista. Dicho de otro modo, todas las variables explicativas (las estadísticas de destreza) fueron tomadas del FIFA 20, a excepción de la data sobre la posición de juego, que también fue tomada de TransferMarkt, junto con las cotizaciones (ver Figura $\mathrm{N}^{\circ} 6$ ).

\section{Figura $\mathbf{N}^{\circ} \mathbf{6}$}

Posiciones de juego según TransferMarkt.

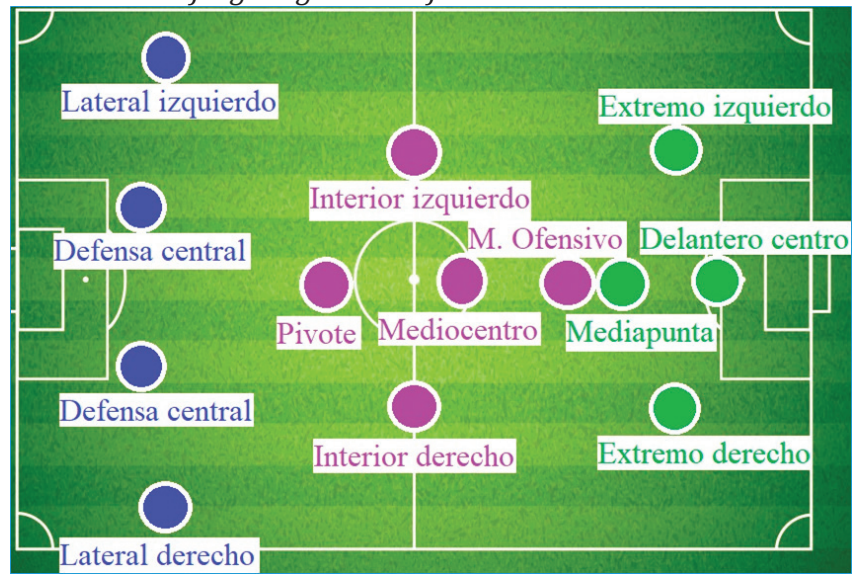

Fuente: Elaboración propia. 
A pesar de que no se incluyeron a los arqueros en nuestra muestra, teníamos doce posiciones de juego. ¿Cómo es esto posible si solo puede haber diez futbolistas en el campo aparte del arquero? Sucede que hablar de "defensas" (en azul), "volantes" (en morado) y "delanteros" (en verde) es una sobre-simplificación de la cantidad de posiciones de juego que existen. Incluso en el argot profesional hay discrepancias entre cuántas posiciones hay y cómo se deben llamar éstas (Corbeto). La figura anterior explica gráficamente dónde se ubican las posiciones de juego según TransferMarkt, que son las que utilizamos para nuestro modelo.

Con respecto a la lista de estadísticas de destreza que tienen los jugadores en FIFA 20, contamos 30, más la Altura, Peso y Edad, y 11 de las 12 posiciones de juego, tenemos 44 variables explicativas en total, las cuales se listan en la Tabla $\mathrm{N}^{\circ} 2$, junto con los resultados de correr la regresión lineal múltiple en el software econométrico "Gretl", con desviaciones típicas robustas ante heteroscedasticidad.

La cuarta columna, "Estad T", resulta de dividir los valores de la segunda y tercera columna "Coeficiente/Desv. Típ.", y es el estadístico de prueba que se distribuye en una T de Student con "n-k-1" grados de libertad, donde "n" es el número de observaciones (280 jugadores analizados) y "k" es el número de variables explicativas sin incluir a la constante (44), es decir, con 235 grados de libertad para el caso de nuestro modelo (Hill et. al., págs. 120-124).

La cantidad de probabilidad acumulada en la cola de la distribución (izquierda o derecha según el signo del estadístico de prueba) se muestra en la quinta columna "Valor P". Se indica con un asterisco $\left(^{*}\right)$ si el coeficiente de la variable es estadísticamente diferente de cero para un nivel de significancia del 10\% ( $\mathrm{p}<\alpha=0.1)$, como es el caso de la variable "Equilibrio". Con dos asteriscos $\left({ }^{* *}\right)$ para un nivel de significancia de $5 \%(\mathrm{p}<\alpha=0.05)$, como es el caso de las variables "Compostura" y "Pase Largo". Y con tres asteriscos $\left({ }^{* * *}\right)$ para un nivel de significancia de $1 \%(\mathrm{p}<\alpha=0.01)$, como es el caso de las variables “Constante”, "Edad”, “Anticipación” y "Fuerza”.

Cabe señalar que hacer robustas a las desviaciones típicas (o "errores estándar") ante heteroscedasticidad cambia el valor de las mismas, y al ser el denominador en el ratio de la prueba estadística, puede alterar el nivel de significancia de los coeficientes de las variables. Tal es el caso de "Intercepción”, que sin los errores estándar robustos sí hubiese sido marcada con un 
asterisco $\left(^{*}\right)$, mientras que "Equilibrio" hubiese dejado de ser significativa al $10 \%(\alpha=0.1)$.

\section{TablaN $^{\circ} 2$}

Resultados de la regresión líneal múltiple: Cotizaciones de los jugadores en TransferMarkt en función de sus destrezas según FIFA20.

\begin{tabular}{|c|c|c|c|c|}
\hline VARIABLE & COEFICIENTE & DESV.TÍP. & ESTAD T & VALOR P \\
\hline const & -361.814 & 80.9804 & -4.468 & $0.00001^{* * *}$ \\
\hline ALTURA__cms & 0.550973 & 0.38672 & 1.425 & 0.15556 \\
\hline PESO__kg & -0.209606 & 0.20383 & -1.028 & 0.30484 \\
\hline EDAD & -2.33304 & 0.41331 & -5.645 & $<0.00001^{* * *}$ \\
\hline Pivote & -12.0149 & 15.0589 & -0.798 & 0.42576 \\
\hline Mediocentro_ofen & -9.39915 & 15.2545 & -0.616 & 0.53839 \\
\hline Mediocentro & -15.9205 & 14.5941 & -1.091 & 0.27644 \\
\hline Mediapunta & -7.77160 & 18.8302 & -0.413 & 0.68019 \\
\hline Lateral_izquierd & -3.75755 & 15.2790 & -0.246 & 0.80595 \\
\hline Lateral_derecho & -9.09799 & 14.9474 & -0.609 & 0.54333 \\
\hline Extremo_izquierd & -7.76741 & 15.1039 & -0.514 & 0.60755 \\
\hline Extremo_derecho & -11.0749 & 15.2706 & -0.725 & 0.46902 \\
\hline Delantero_centro & -17.3880 & 16.8778 & -1.030 & 0.30396 \\
\hline Defensa_central & -2.49940 & 15.6289 & -0.160 & 0.87308 \\
\hline Interior_izquier & -12.2863 & 15.3125 & -0.802 & 0.42315 \\
\hline ZURDO & -0.331473 & 3.06764 & -0.108 & 0.91404 \\
\hline CONTROL_DE_BALON & -0.109263 & 0.41367 & -0.264 & 0.79191 \\
\hline REGATES & -0.279060 & 0.24938 & -1.119 & 0.26427 \\
\hline MARCAJE & -0.193019 & 0.23453 & -0.823 & 0.41134 \\
\hline ENTRADAS & 0.279122 & 0.29369 & 0.950 & 0.34288 \\
\hline ROBOS & 0.002926 & 0.33222 & 0.009 & 0.99298 \\
\hline AGRESIVIDAD & 0.031240 & 0.13475 & 0.232 & 0.81687 \\
\hline ANTICIPACION & 2.70568 & 0.44122 & 6.132 & $<0.00001^{* * *}$ \\
\hline POSICION_ATAQUE & 0.130430 & 0.23514 & 0.555 & 0.57964 \\
\hline INTERCEPCION & -0.351809 & 0.27637 & -1.273 & 0.20428 \\
\hline VISION & 0.126880 & 0.20341 & 0.624 & 0.53338 \\
\hline COMPOSTURA & 0.620703 & 0.30440 & 2.039 & $0.04256^{* *}$ \\
\hline CENTROS & 0.009056 & 0.150249 & 0.060 & 0.95199 \\
\hline PASE_CORTO & 0.158760 & 0.392059 & 0.405 & 0.68589 \\
\hline PASE_LARGO & 0.551579 & 0.277096 & 1.991 & $0.04769 * *$ \\
\hline ACELERACION & 0.215135 & 0.355431 & 0.605 & 0.54558 \\
\hline RESISTENCIA & 0.045289 & 0.184144 & 0.246 & 0.80594 \\
\hline FUERZA & 0.731590 & 0.252302 & 2.900 & $0.00409 * * *$ \\
\hline EQUILIBRIO & 0.387894 & 0.227257 & 1.707 & $0.08917^{*}$ \\
\hline VELOCIDAD & 0.154716 & 0.281428 & 0.550 & 0.58301 \\
\hline AGILIDAD & 0.087354 & 0.206524 & 0.423 & 0.67270 \\
\hline SALTO & -0.145973 & 0.104692 & -1.394 & 0.16454 \\
\hline CABEZAZOS & -0.022382 & 0.216246 & -0.104 & 0.91765 \\
\hline POTENCIA_DE_TIRO & -0.051717 & 0.191871 & -0.270 & 0.78775 \\
\hline DEFINICION & -0.037170 & 0.209293 & -0.178 & 0.85919 \\
\hline TIROS_LEJANOS & 0.135011 & 0.173758 & 0.777 & 0.43794 \\
\hline EFECTO & -0.099951 & 0.158416 & -0.631 & 0.52869 \\
\hline PRECISION_DE_FALTA & -0.137122 & 0.118667 & -1.156 & 0.24905 \\
\hline PENALTIS & 0.112090 & 0.106054 & 1.057 & 0.29164 \\
\hline VOLEAS & -0.013998 & 0.136360 & -0.103 & 0.91833 \\
\hline
\end{tabular}

(Fuente: Elaboración propia) 


\subsection{Sobre la variable "Posición".}

La data original contenía dos variables cualitativas, "Pie" y "Posición". La primera era binomial, sus valores eran "Izquierdo" o "Derecho", por lo que fácilmente se convirtió en una variable dicotómica "Zurdo", de modo que los que tenían valor "Izquierdo" pasen a tener un "1" (de "sí” o "verdadero") y los que tenían valor "Derecho" pasen a tener un "0" (de "no" o "falso").

Por otro lado, la variable "Posición" era una cualitativa multinomial, pues tenía hasta 12 categorías: (1) Pivote, (2) Mediocentro ofensivo, (3) Mediocentro, (4) Mediapunta (5) Lateral izquierdo, (6) Lateral derecho, (7) Extremo izquierdo, (8) Extremo derecho, (9) Delantero centro, (10) Defensa central, (11) Interior izquierdo e (12) Interior derecho (como se explicó al inicio de esta sección). Es así que las 11 primeras categorías fueron convertidas en variables dicotómicas o "dummies", dejando a la última como base para evitar el problema de co-linearidad perfecta: Cuando todas las dummies adopten el valor de cero, quedará sobreentendido que la variable dicotómica base hubiese adoptado el valor de uno (Hill et. al., págs. 153-156).

Ninguna de las 11 dummies asociadas a "Posición" fueron estadísticamente significativas (ninguna de las que está de color gris en la tabla tenía algún asterisco). Esto nos hizo preguntar si la variable "Posición" en sí era relevante o no para el modelo. El problema de sobre-ajuste ("over-fitting") es opuesto al de sesgo de variable omitida ("ommited variable bias"): No solo vuelven al modelo innecesariamente más complejo, sino que suelen inflar los errores estándar de los coeficientes, volviendo a algunas variables no-significativas cuando de no ser por ellas sí lo hubiesen sido (Hill et. al., págs. 150-152).

Corrimos entonces otra regresión lineal con las mismas variables (tanto dependiente como independientes), salvo por las 11 dicotómicas asociadas a "Posición". El coeficiente de determinación " $\mathrm{R}^{\wedge} 2$ " obviamente bajó su porcentaje de explicación, de $70.31 \%$ a 68.88\%, debido a la menor cantidad de regresores incluidos. Es por ello que el verdadero indicador que se debe utilizar para comparar aquí es el "R^2 corregido" (o "R-cuadrado ajustado"), que sí mide la eficiencia del modelo al sopesar la pérdida de poder explicativo con la ganancia de sencillez (Triola, pág. 544):

(1) $R^{2}$ corregido $=1-\frac{(n-1)}{(n-k-1)}\left(1-R^{2}\right)$ 
Donde " $n$ " es el número de observaciones y "k" es el número de variables sin incluir a la constante. En la fórmula se puede apreciar que, incluso para una regresión lineal simple $(\mathrm{k}=1), \mathrm{R}^{\wedge} 2 \neq \mathrm{R}^{\wedge} 2$ corregido. Utilizando este indicador en vez, aún podemos ver que el $\mathrm{R}^{\wedge} 2$ ajustado baja de $64.75 \%$ a $64.70 \%$ cuando quitamos a todas las dummies asociadas a la variable multinomial "Posición", de lo que concluimos que, si bien ninguna de ellas es significativa individualmente, colectivamente sí lo son.

Sin embargo, se observa que cada uno de los coeficientes de las variables dicotómicas para "Posición" ingresa al modelo con signo negativo, que es contrario al que se esperaba. Esto podría deberse a que aún persiste una alta co-linearidad (a pesar de haberla mitigado poniendo a una de las dummies como base para las demás), así como un problema de sobreajuste (debido a la gran cantidad de destrezas tomadas de FIFA 20).

\subsection{Sobre la variable omitida "Edad^2"}

La significancia de la posición del futbolista dentro del campo se condice con el pensamiento común de que ésta sí tiene un efecto sobre su cotización, favoreciendo a los que juegan de delantero (y perjudicando a los que juegan de defensa). Esto es algo común a todos los deportes colectivos, lo que demostraría que hacer anotaciones sí es más importante que evitar que te las hagan (o al menos que el mercado lo valora más). Ante esto, uno podría preguntarse qué incentivos tienen los defensas para no querer ser también delanteros, y las respuestas son varias: menos competencia por el puesto, menor escrutinio por parte de la opinión pública, y una mayor duración de la carrera profesional, dado que las destrezas que esta posición requiere menguan menos con la edad: Anticipación, Compostura, Pase largo, Fuerza y Equilibrio, que coinciden con ser las que fueron significativas en nuestro modelo.

El otro regresor que es significativo incluso al 1\% ( $p<\alpha=0.01)$, es "Edad", una variable cuantitativa que aquí se incluye solo de forma lineal, cuando la teoría y el sentido común nos sugieren que deberíamos incluir también un término cuadrático para darle curvatura a la relación que existe entre la cotización de un jugador y sus años cumplidos. Ya en el párrafo anterior advertíamos que ciertas destrezas sí menguan con la edad, tales como Aceleración, Resistencia, Agilidad y Velocidad, habilidades particularmente importantes 
para los futbolistas que juegan en posiciones ofensivas y que coinciden con ser variables poco significativas en nuestro modelo.

$\mathrm{Al}$ correr una regresión con las mismas variables explicativas más el componente cuadrático ("Edad^2"), vimos que este último no solo no era individualmente significativo, sino que colectivamente hacía disminuir el R-cuadrado ajustado (de 64.75\% a 64.73\%), en adición a aumentar los valores de los Criterios de Información de Akaike ("AIC"), Bayesiano de Shwarz ("BIC") y de Hannan-Quinn ("HQC"), cuyas fórmulas y valores para cada modelo se muestran en la Tabla $\mathrm{N}^{\circ} 3$.

\section{Tabla $\mathbf{N}^{\circ} 3$}

Comparación entre modelos según Criterios de Información.

\begin{tabular}{llll}
\hline Fórmula & Sin Edad^2 & Con Edad^2 & Variación \\
\hline $\mathrm{AIC}=2 * \mathrm{k}-2 * \operatorname{Ln}(\mathrm{L})$ & $2,399.15$ & $2,400.13$ & +0.98 \\
$\mathrm{BIC}=\mathrm{k}^{*} \operatorname{Ln}(\mathrm{n})-2 * \operatorname{Ln}(\mathrm{L})$ & $2,562.72$ & $2,567.33$ & +4.61 \\
$\mathrm{HQC}=2 * \mathrm{k}^{*} \operatorname{Ln}(\operatorname{Ln}(\mathrm{n}))-2 * \mathrm{~L}$ & $2,464.76$ & $2,467.20$ & +2.44 \\
\hline
\end{tabular}

Fuente: Wikipedia (AIC, BIC, HQC)

Los tres criterios son distintas formas de medir la cantidad de información perdida, por lo que los tres se interpretan de la misma manera: El mejor modelo es el que menor valor tenga, donde "L" es el máximo valor de la función de verosimilitud, "k" es el número de parámetros (variables más intercepto) y " $n$ " el tamaño de la muestra. Como $\operatorname{Ln}(\mathrm{n})>2$ para todo $\mathrm{n}>\mathrm{e}^{\wedge} 2=7.389$, el BIC hace una mayor penalización por sobre-ajuste del modelo (por incluir parámetros innecesarios) que el AIC (+4.61 vs. +0.98). El HQC es el indicador más consistente, mientras el AIC es el más eficiente (Claeskens \& Hort, 2008).

Optamos pues, por excluir al término cuadrático para la variable "Edad" y nos quedamos solo con su término lineal. Pero, ¿por qué es que el término cuadrático no fue estadísticamente significativo? Si bien es cierto que muchas destrezas importantes para ser un buen futbolista van menguando con los años, sucede también que, siendo la Premier League una liga de élite, es raro que los futbolistas sigan jugando en ella mucho tiempo después de haber alcanzado su máximo rendimiento (el vértice de la parábola). En la mayoría de casos, la alta competencia entre equipos (y entre jugadores de un mismo equipo), les obliga a migrar a otras ligas menos competitivas, sino a retirarse del fútbol por completo. 
De hecho, el periodista Aaron Bower publicó en el 2013 una lista de los diez jugadores más veteranos en la historia de la Premier hasta ese entonces. Incluía muchos nombres conocidos, como el de Ryan Giggs ( $1^{\circ}$ con 39 años) y Paul Scholes ( $2^{\circ}$ con 38), ambos del Manchester United, Dennis Bergkamp del Arsenal ( $3^{\circ}$ con 36$)$, Jamie Carragher del Liverpool ( $5^{\circ}$ ) y Alan Shearer del Newcastle $\left(6^{\circ}\right)$, ambos con 35 años al momento de su retiro. Todos alcanzaron el status de leyendas en sus respectivos clubes (Bower).

¿Y quiénes son los mejores futbolistas veteranos de la Premier en la actualidad? El portal FootballCritic menciona a Thiago Silva (36) y Olivier Giroud (34), ambos del Chelsea; a Jamie Vardy (33) y Kasper Schmeichel (34), ambos del Leicester; a Hugo Lloris del Tottenham (34) y a Fernandinho del Manchester City (35). Qué duda cabe que todos los listados tienen todavía para rato (FootballCritic). Mención honrosa para Edison Cavani del Manchester United (33).

\section{Resultados y Discusión.}

Habiendo aceptado el modelo con las desviaciones típicas robustas ante heteroscedasticidad, con las 11 dummies para las posiciones de los jugadores dentro del campo, y sin el término cuadrático para la edad, volvamos a observar la Figura $\mathrm{N}^{\circ} 1$ de la introducción y pasemos a interpretar las implicancias del modelo de la siguiente manera:

En principio, aquellos jugadores cuya cotización sea superior al precio que deberían de tener según nuestro modelo, los puntos que están por encima de la línea roja, están sobre-valuados ("over-priced"). A nivel de clubes, comprarlos sería un mal negocio y venderlos uno bueno. Por contraposición, aquellos jugadores cuya cotización sea inferior al precio que deberían de tener según nuestro modelo, los puntos que están por debajo de la línea roja, están sub-estimados ("under-estimated"). A nivel de clubes, venderlos sería un mal negocio y comprarlos -o retenerlos- uno bueno.

Esto claro, asumiendo que el videojuego FIFA 20 logra asignar correctamente el valor de los futbolistas de la Premier League, independientemente de las cotizaciones que se muestran en la página web de TransferMarkt. Aquí fue clave tomar a los 14 jugadores de cada club con más minutos en la presente temporada, que tuviesen la suficiente exposición como para que FIFA 20 pudiera asignarles estadísticas de destreza a partir de haberlos 
observado verdaderamente, y no simplemente asignar valores de manera semi-aleatoria según su cotización.

Obsérvese la esquina superior derecha de la Figura $\mathrm{N}^{\circ} 7$, contiene cinco valores extremos ("outliers"). El punto más alto corresponde a Raheem Sterling del Manchester City: Con una cotización de €160 millones en TransferMarkt, nuestro modelo solo le asigna un valor de €94.69 millones a partir de sus habilidades según FIFA20. Los cuatro puntos debajo corresponden a cuatro jugadores que tienen la misma cotización de $€ 150$ millones, pero que nuestro modelo les asigna los siguientes valores, de izquierda a derecha: €92.70 millones para Kevin de Bruyne (también del Manchester City), €94.94 millones para Harry Kane (del Tottenham), $€ 97.26$ millones para Sadio Mané y €99.18 millones para Mohamed Salah, ambos del Liverpool. Los tres últimos tienen un valor de acuerdo a nuestro modelo (basado en las estadísticas de FIFA 20) que supera al del jugador con la mayor cotización en TransferMarkt.

\section{Figura $\mathbf{N}^{\circ} 7$}

Cotizaciones en TransferMarkt (" $y$ ", eje vertical) en función lineal del valor del jugador según sus estadísticas en FIFA 20 (" $y^{\wedge ",}$ eje horizontal)

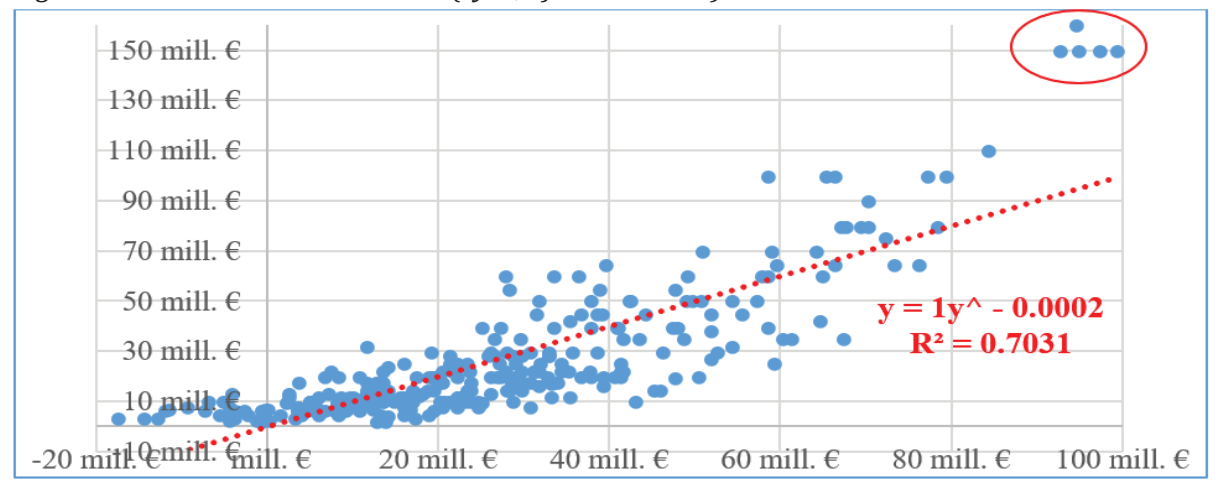

Fuente: Elaboración propia.

Estos cinco futbolistas se encuentran en un "clúster" muy apartado de sus demás colegas: Quien les sigue, Trent Alexander-Arnold (también del Liverpool, con una cotización de €110 millones y un valor de €84.39 millones), está más cerca al resto de jugadores que a este grupo de valores extremos. Lo que es más importante, es que difícilmente alguien quiera 
recomendar vender a alguno de estos cinco futbolistas por estar sobre-valuados aun después de haber observado el gráfico anterior.

Surge entonces la necesidad de hacer algún ajuste para esta línea recta divisoria, que con pendiente igual a 1 y con intercepto estadísticamente igual a 0 , el coeficiente de determinación es de solo 70.31\%. Al trazar una línea de tendencia exponencial, por ejemplo, el porcentaje de variación que sí logra ser explicado por el modelo sube a 73.12\%, y los cinco futbolistas mejor cotizados de la Premier pasan de estar sobre-valuados a estar sub-estimados (ver Figura Nº).

\section{Figura $\mathbf{N}^{\circ} \mathbf{8}$}

Cotizaciones en TransferMarkt (y) en función exponencial del valor del jugador según sus estadísticas en FIFA $20\left(y^{\wedge}\right)$.

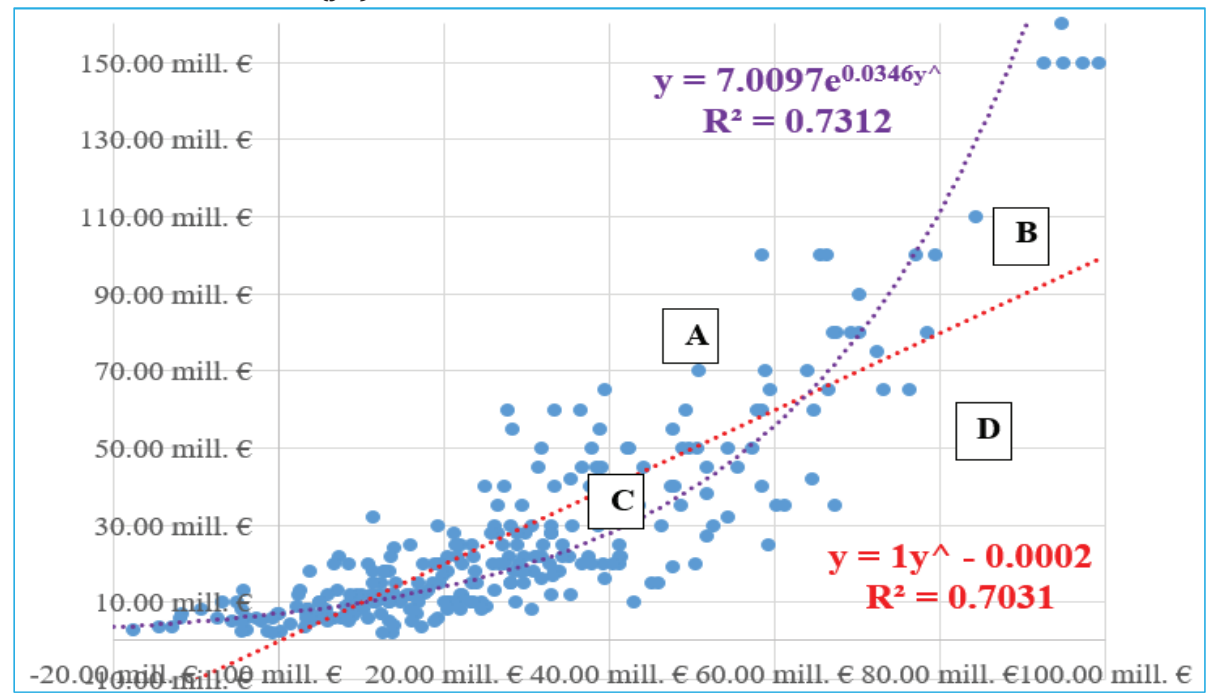

Fuente: Elaboración propia.

De hecho, si conservamos a las dos líneas de tendencia en la Figura $\mathrm{N}^{\circ} 8$, la lineal y la exponencial, vemos que entre ambas dividen el plano en cuatro áreas o regiones. La región "B" contiene a los jugadores que están sobre-valuados de acuerdo al criterio lineal, pero sub-estimados según el criterio exponencial. En la región " $\mathrm{C}$ " sucede lo opuesto, contiene a los jugadores que están sobre-valuados de acuerdo al criterio exponencial, pero sub-estimados según el criterio lineal. Sin embargo, el mismo hecho de que los jugadores estén sobre-valuados o sub-estimados de acuerdo a un criterio u otro, nos lleva a concluir que aquellos futbolistas que yacen en alguna de estas dos áreas, "B" 
y “C”, están solo moderadamente sobre-valuados o sub-estimados (según la perspectiva de donde se les mire). En cambio, la región " $\mathrm{A}$ ” contiene a los jugadores que están sobre-valuados de acuerdo a los criterios lineal y exponencial a la vez, y que por tanto estarían seriamente sobre-valuados. En la región "D" ocurre lo contrario, contiene a los jugadores que están sub-estimados tanto según el criterio lineal como el exponencial, con lo cual estarían seriamente sub-estimados.

Si bien el modelo exponencial corrige en cierta manera las interpretaciones del modelo lineal, que señalaba a ese grupo de cinco "outliers" como severamente sobre-valuados y que había que vender con urgencia porque eran malas inversiones, el modelo exponencial también exagera en el sentido opuesto, al señalar que estos jugadores con cotizaciones superiores a los €150 millones estarían siendo sub-estimados más bien. Surge pues, la necesidad de una tercera línea de tendencia, una cuadrática (o polinómica de grado 2) que, como se aprecia en Figura $N^{\circ}$, yace en su mayoría entre la exponencial y la lineal, a la vez que atraviesa mejor a todas las observaciones, como se constata en el coeficiente de determinación, de 82.42\%.

\section{Figura $\mathbf{N}^{\circ} \mathbf{9}$}

Cotizaciones en TransferMarkt (y) en función cuadrática del valor del jugador según sus estadísticas en FIFA $20\left(y^{\wedge}\right)$.

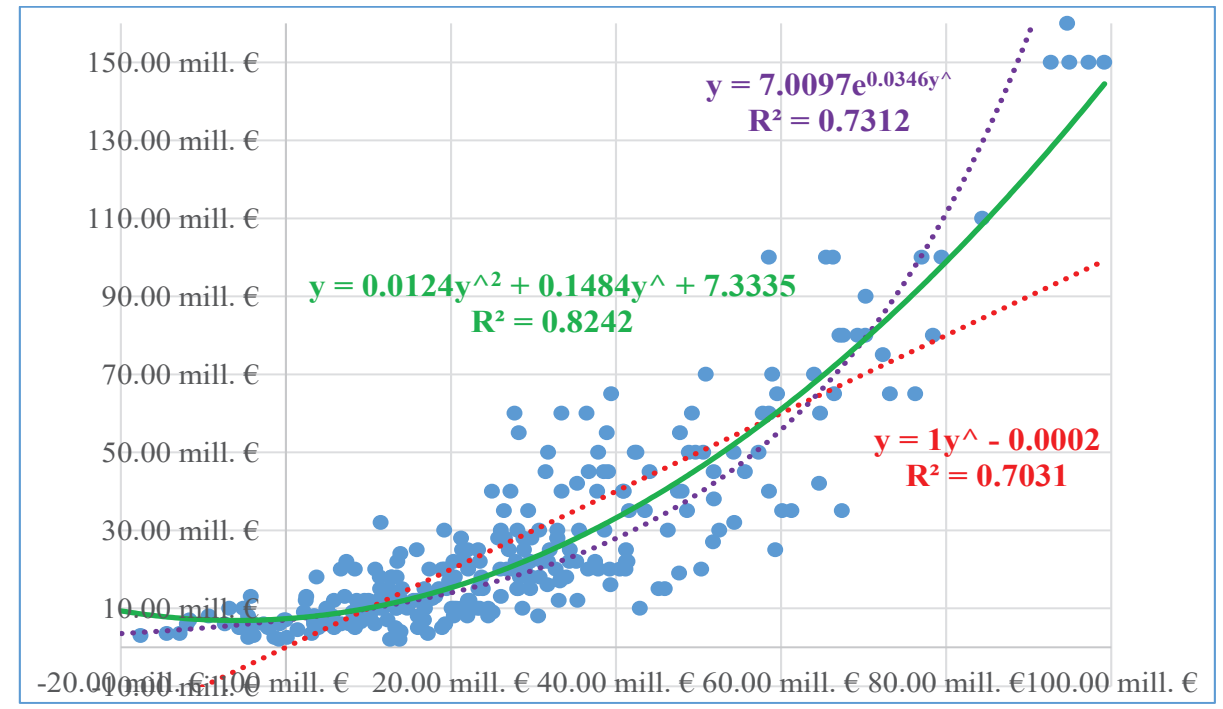

Fuente: Elaboración propia. 


\section{1.¿Por qué la función lineal no es la de mayor bondad de ajuste?}

Podemos ver que tanto la línea de tendencia exponencial $\left(\mathrm{R}^{2}=0.7312\right)$ y sobre todo la cuadrática $\left(\mathrm{R}^{2}=0.8242\right)$ tienen una mayor bondad de ajuste que la lineal $\left(R^{2}=0.7031\right)$, que es la forma funcional con la que el modelo econométrico fue concebido originalmente. Esto se debe a la restricción del número de jugadores en el campo que existe en los deportes colectivos, que hace que uno no pueda intercambiar calidad por cantidad, volviendo mucho más valioso a un jugador que es solo un poco mejor que otro, es decir, las destrezas en los trabajos con restricción de capacidad se recompensan en el margen de manera creciente (por ejemplo, se puede cambiar a un cajero rápido por dos o tres cajeros lentos que juntos logren atender a más clientes, pero no se puede cambiar a un delantero bueno por tres delanteros malos porque solo puedes poner a once futbolistas en el campo).

Este razonamiento podría ser también el que haya llevado a una "inflación" en el salario de los deportistas profesionales varias veces mayor al del resto de trabajadores: Entre 1985 y 2012, el ingreso mediano de hogares estadounidenses se duplicó, manteniéndose a la par del nivel general de precios, mientras que el de los beisbolistas se multiplicó por nueve y el de los basquetbolistas, por dieciséis (Peltz). Por tanto, es equivocado atribuir la híper-inflación que ha habido en el salario de los atletas profesionales meramente a las inyecciones de liquidez (dinero puesto en circulación) en años recientes por parte de bancos centrales como el de Inglaterra y el de la Unión Europea (Black).

Otra explicación plausible para el aumento salarial extra que tuvo la industria del deporte es el de la "inflación" que hubo en las audiencias con los cambios tecnológicos (que pasó de los miles que cabían en los estadios, a los millones que veían los juegos por televisión de antena, y a los miles de millones que los ven ahora alrededor del mundo por señal de cable o satelital), así como a la sindicalización que se logró a través de agentes representantes ("managers") que compensaron el poder de negociación de los clubes, que se coludían en un "acuerdo de caballeros" para no subastarse entre sí a los jugadores (Stephenson).

En el caso particular del fútbol, se puede identificar un cambio en la trayectoria de los salarios a partir del año 1995, cuando una corte europea 
falló en el "Caso Bosman" a favor de que los jugadores cuyo contrato hubiese vencido pudiesen cambiar de equipo sin necesidad de realizar un pago por el traspaso, con lo cual los clubes utilizaron ese presupuesto ahorrado para pagar mayores salarios a sus planteles (Badenhausen). Sin perjuicio de lo anterior, los millonarios ingresos por los derechos de transmisión de los partidos por televisión inyectaron a los clubes de fondos disponibles para el pago de transferencias que no fueron acompañados por un aumento en la oferta de jugadores de clase mundial, generando así una presión inflacionaria en las cotizaciones (McLeod).

\section{2. ¿Qué clubes tuvieron las plantillas más sobre-valuadas y cómo les fue?}

Así pues, nos quedamos con la forma funcional cuadrática por tener el mayor coeficiente de determinación, en lo que es de facto una regresión en dos etapas: Primero, una regresión lineal múltiple (que incluye a todos los indicadores de destreza en FIFA 20), y a partir de ésta, una regresión nolineal simple (que admite que, por la restricción de capacidad, se valoran las destrezas en el margen de forma creciente).

Lo que hicimos a continuación fue sumar en cada club las cotizaciones según TransferMarkt de los catorce futbolistas con más minutos en el campo de juego y aparte, sumar los valores que deberían de tener esos mismos jugadores de acuerdo a nuestro modelo cuadrático, para ver a partir de sus diferencias, qué clubes estarían teniendo en general plantillas sobre-valuadas (si la discrepancia es positiva, es decir, si las cotizaciones superan a nuestras valoraciones) y también claro, qué clubes tendrían planteles sub-estimados (si la discrepancia es negativa, esto es, si nuestras valoraciones superan a las cotizaciones), comparándolo a su vez, contra la tabla de posiciones al final de la Premier League 20192020 (recuérdese que la data fue extraída durante la para que hubo en el campeonato por la pandemia). Los resultados se muestran en la Tabla $\mathrm{N}^{\circ} 4$.

Por un lado, es una grata sorpresa ver que el equipo que salió campeón, el Liverpool, lo hizo teniendo un plantel sub-estimado, demostrando la inteligencia de sus dirigentes y comando técnico para contratar (y dejar ir) jugadores, a pesar de tener el segundo plantel más caro del 
campeonato (€987 milones), incluidos Mohamed Salah, Sadio Mané y Trent Alexander-Arnold, que estaban moderadamente sobre-valuados incluso para la línea de tendencia cuadrática (ver Figura $\mathrm{N}^{\circ}$ ). La otra sorpresa grata es la del Tottenham, que también ocupó un puesto de clasificación a competencias europeas, teniendo la segunda plantilla más sub-estimada del campeonato (solo después del Newcastle), pues debería de valer unos €73 millones más.

\section{Tabla $\mathbf{N}^{\circ} \mathbf{4}$}

Discrepancias entre las cotizaciones de los planteles de la Premier League 2019-20 y sus valores según nuestro modelo (en millones de €)

\begin{tabular}{|c|c|c|c|c|}
\hline Equipo & $\Sigma$ Cotizaciones & $\Sigma$ Valor según modelo & Discrepancia & Interpretación \\
\hline 1. Liverpool & 987.0 & 996.68 & -9.7 & subestimado \\
\hline 2. Man. City & 990.0 & 892.81 & 97.2 & sobrevaluado \\
\hline 3. Man. United & 581.0 & 528.83 & 52.2 & sobrevaluado \\
\hline 4. Chelsea & 579.0 & 427.12 & 151.9 & sobrevaluado \\
\hline 5. Leicester & 436.0 & 340.32 & 95.7 & sobrevaluado \\
\hline 6. Tottenham & 710.0 & 783.38 & -73.4 & subestimado \\
\hline 7. Wolverhampton & 324.0 & 322.10 & 1.9 & sobrevaluado \\
\hline 8. Arsenal & 509.0 & 451.88 & 57.1 & sobrevaluado \\
\hline 9. Sheffield & 122.0 & 177.27 & -55.3 & subestimado \\
\hline 10. Burnley & 155.0 & 189.17 & -34.2 & subestimado \\
\hline 11. Southampton & 178.5 & 169.05 & 9.4 & sobrevaluado \\
\hline 12. Everton & 370.0 & 366.97 & 3.0 & sobrevaluado \\
\hline 13. Newcastle & 217.0 & 292.80 & -75.8 & subestimado \\
\hline 14. Crystal Palace & 174.0 & 205.00 & -31.0 & subestimado \\
\hline 15. Brighton & 167.5 & 190.51 & -23.0 & subestimado \\
\hline 16. West Ham & 287.0 & 285.37 & 1.6 & sobrevaluado \\
\hline 17. Aston Villa & 222.0 & 243.83 & -21.8 & subestimado \\
\hline 18. Bournemouth & 267.5 & 273.33 & -5.8 & subestimado \\
\hline 19. Watford & 167.5 & 235.26 & -67.8 & subestimado \\
\hline 20. Norwich & 120.5 & 175.45 & -54.9 & subestimado \\
\hline
\end{tabular}

Fuente: Elaboración propia 
Por otro lado, es una sorpresa no-grata ver que el equipo que salió sub-campeón, el Manchester City, lo hizo teniendo el segundo plantel más sobre-valuado (+€97.2 millones) y el más caro del campeonato (€990 millones), aunque los valores extremos de Raheem Sterling y Kevin de Bruyne influyeron mucho en estas estadísticas. La otra sorpresa no-grata fue la del Chelsea, que también ocupó un puesto de clasificación a competencias europeas, teniendo la plantilla más sobre-valuada del campeonato tanto en términos absolutos (+€151.9 millones) como relativos, ya que esta discrepancia representa un $26 \%$ con respecto a la suma de sus cotizaciones.

En general, los resultados han sido decepcionantes y se resumen en los desenlaces para los equipos Leicester y Watford, quienes como en el cuento de Charles Dickens, narran "la historia de dos ciudades": Por un lado, el Leicester, tuvo al tercer plantel más sobre-valuado (+€95.7 millones) y aun así logró clasificar a una competencia europea. Por el otro lado, el Watford, tuvo al tercer plantel más sub-estimado (-€67.8 millones) y aun así descendió a la segunda división (EFL Championship).

Considerando que el coeficiente de determinación $\left(\mathrm{R}^{2}\right)$ es el literalmente el cuadrado del coeficiente de correlación (R) en las regresiones lineales simples, podemos ver en la Figura $\mathrm{N}^{\circ} 10$ que existe más bien una correlación positiva entre cuan alta es la ubicación en la tabla de posiciones y cuán sobre-valuada está tu plantilla $(+58 \%)$, lo que a su vez se reafirma en el hecho de que la ubicación en la tabla de posiciones se correlaciona más positivamente con las cotizaciones de TransferMarkt (+79\%) que con las valorizaciones según nuestro modelo (+73\%), construido a partir de las estadísticas de destreza en FIFA 20. 


\section{Figura $\mathbf{N}^{\circ} \mathbf{1 0}$}

Relación entre la ubicación en la tabla de posiciones (z) con las cotizaciones de los planteles de la Premier League 2019-20 (s), sus valores según nuestro modelo ( $t$ ) y las discrepancias entre ambos (u), en millones de euros.

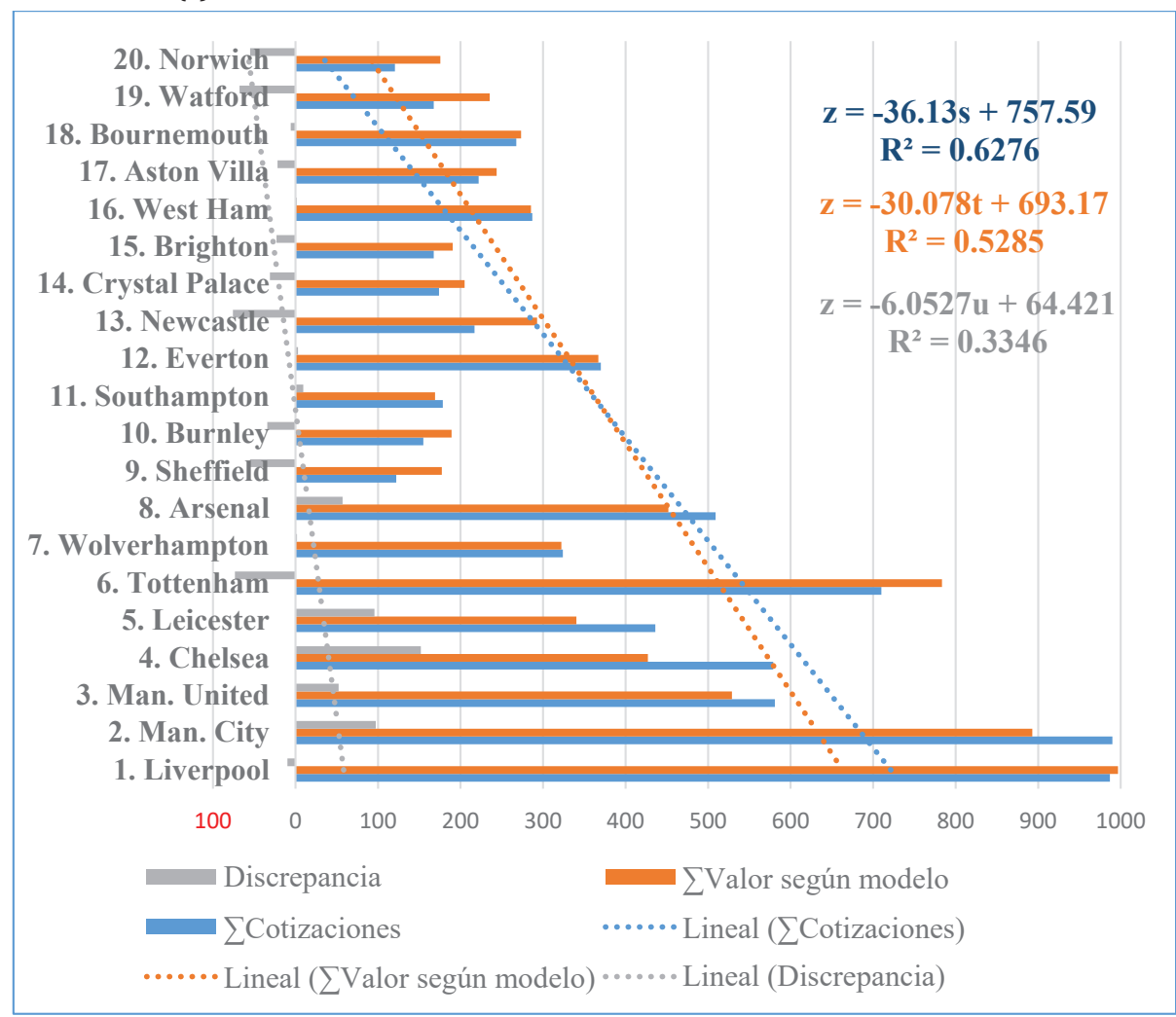

Fuente: Elaboración propia

\section{Reflexiones / Conclusiones / Apreciaciones Finales}

En la deliberación sobre la especificación del modelo econométrico, se terminó incluyendo a la variable multinomial "Posición" a través de sus once regresores binarios, porque conjuntamente lograban aumentar el R-cuadrado corregido; y excluyendo al término cuadrático de la variable "Edad" porque disminuía este mismo indicador, a la vez que aumentaba los distintos criterios de información. 
Sobre lo primero, las dummies tal como estaban solo afectaban a la constante de la regresión, por lo que sería interesante observar si las interacciones con las habilidades son estadísticamente significativas, afectando las "pendientes" de cada variable, lo que de facto implica hacer un modelo econométrico para cada puesto. Esto considerando que nuestra experiencia y conocimientos de este deporte nos dicen que las destrezas se requieren en distintas proporciones según la posición que ocupes en el campo de juego.

Sobre lo segundo, el hecho de que no se llegue a reflejar el declive en la cotización de los futbolistas con el paso de los años se debería a que el total de la muestra se tomó de la Premier League, cuya exigencia es tal que obliga a los jugadores a retirarse a otras de menor competencia (sino del fútbol mismo) cuando ya no están al tope de su rendimiento. Sería interesante pues, observar si el complemento “Edad^2" sí logra ser significativo en ligas de menor élite (con lo que sí se identificaría una curvatura en las cotizaciones).

Luego, en el análisis, vimos que las cotizaciones de los jugadores según la página web TransferMarkt ("y") y las valorizaciones de los mismos de acuerdo sus destrezas en el videojuego FIFA 20 ( “ $\mathrm{y}^{\wedge}$ ”) están altamente correlacionadas (+84\%), y que a un nivel colectivo, ambos criterios se correlacionan de manera positiva con las casillas que los clubes ocuparon en la tabla de posiciones al término de la Premier League 2019-20. Todo esto es consistente con el sentido común: Los jugadores más habilidosos son los que más dinero valen porque son los que le consiguen mejores resultados deportivos a los clubes que pertenecen.

Contrario a lo que esperábamos encontrar, sin embargo, los equipos cuya plantilla estaba más sobre-valuada coincidieron con ser los que consiguieron ocupar las mejores posiciones. Esto se debería a que todos los clubes de la Premier League estarían comprando con inteligencia, de modo que marginalmente no esté premiando tanto a los clubes que consigan una mayor eficiencia en el gasto de contrataciones (ni castigando tanto a los que no).

Sería interesante hacer un modelo más extensivo, que comprenda a las demás ligas de fútbol europeo, para encontrar mayores primas por comprar con inteligencia (como serían los casos del Borussia Dortmund 
de Michael Zorc en Alemania y el Sevilla de Ramón Rodríguez en España), así como para ver qué campeonatos en general están sobre-valuados y sub-estimados debido a sus niveles de eficiencia en el gasto de contrataciones.

Mientras tanto, salvo escasas excepciones, se vendría cumpliendo el refrán popular de "billetera mata galán", un dicho coloquial que originalmente se utiliza cuando una persona con dos pretendientes termina prefiriendo al más adinerado por encima del que le es más atento y gentil. En la Premier League también estaría sucediendo algo similar, pues contrariamente a lo señalado en las románticas columnas de Jorge Barraza (ver sección 3), no se estaría premiando tanto a los clubes que fueron más eficientes en el gasto para armar una plantilla sub-estimada, sino a los más poderosos financieramente, que pueden contratar (y retener) a los jugadores más costosos, así estén sobre-valuados.

Ejemplo de ello fue el Newcastle, que en el 2019-20 terminó el campeonato ocupando puesto de la mitad de tabla para abajo, sin pena ni gloria, a pesar de haber tenido el plantel más sub-estimado tanto en términos absolutos como relativos, pues sus 14 jugadores con más minutos hasta el corte por la pandemia sumaban cotizaciones por €217 millones, mientras que nuestro modelo les asignaba un valor de $€ 292$ millones, una diferencia de €76 millones (+35\%).

Sin embargo, no se debe olvidar que el principal propósito de esta investigación no era encontrar una correlación positiva entre la eficiencia del gasto en contrataciones y logros deportivos alcanzados, sino hallar una metodología de valorización de los futbolistas profesionales, similar a las "métricas-sable" con las que Billy Beane y Paul DePodesta revolucionaron la Liga Mayor de Béisbol desde los Oakland Athletics (ver sección 2).

En ese sentido, consideramos que la investigación ha sido un éxito. Véanse por ejemplo los casos de los dos futbolistas más sub-estimados de la Premier League, el del portugués Diogo Jota y el del francés Allan Saint-Maximin. Ambos juegan de extremo izquierdo, pero sus puntos en el gráfico eran los que se situaban más cercanos a la esquina inferior derecha, muy por debajo de todas las líneas de tendencia. Jota entonces estaba cotizado en $€ 35$ millones de acuerdo a TransferMarkt, debía valer €67.38 millones según nuestro modelo econométrico (basado en 
las estadísticas de destreza en FIFA 20) y jugaba en el Wolverhampton Wanderers. Actualmente juega en el Liverpool FC y está cotizado en €40 millones (TransferMarkt [1]). Saint-Miximin estaba cotizado en €25 millones, pero nuestro modelo le asignaba un valor de €59.32 millones. Sigue jugando en el Newcastle, pero su cotización subió a €32 millones (TransferMarkt [2]).

Vemos pues que también se estaría cumpliendo la Hipótesis de Mercados Eficientes de las Finanzas en la industria del fútbol: los jugadores que estaban seriamente sub-estimados tienden a corregir su precio, convergiendo hacia su verdadero valor, y nuestro modelo permitiría identificar quiénes son antes de que lo hagan. Todos los clubes tienen incentivos para utilizar este método de valorización de jugadores en su afán de maximizar la eficiencia de su gasto en contrataciones: los de bajo presupuesto que luchan por la permanencia, como los de presupuesto relativamente alto en el campeonato local que cuando participan en torneos internacionales pasar a ser equipos "chicos". Las únicas limitantes de nuestro modelo son que requiere que los jugadores estén cotizados en la página web de TransferMarkt y que a la vez aparezcan en el videojuego de FIFA 20.

\section{Referencias Bibliográficas}

Badenhausen, Kurt. "The Highest-Paid Athletes Of 1995 Vs. Today". Forbes, 18/09/2015.

Barraza, Jorge [1]. "Arsenal y el encanto del fútbol inglés". El Comercio, 02/08/2020.

Barraza, Jorge [2]. "Lukaku, el tiro por la culata”. El Comercio, 23/08/2020.

Barrio de Mendoza, Diego. "Boxing Day: ¿por qué hay fútbol profesional en Inglaterra al día siguiente de Navidad?". El Comercio, 25/12/2020.

Bascombe, Chris. "Arsenal manager Arsene Wegner is an idol of mine, says revered baseball coach Billy Bean". The Telegraph, 13/10/2011.

Baumer, Benjamin \& Andrew Zimblist. "The Sabermetrics Revolution: Assessing the Growth of Analytics in Baseball". Philadelphia: University of Pennsylvania Press, 2014.

Black, Simon. "No inflation Friday: How sport salaries reveal the true rate of inflation". Sovereign Man, 31/10/2014. 
Bower, Aaron. "Top 10 Veterans in Premier League History". BleacherReport, 28/11/2013.

Canelo, Pedro. "Alianza Lima: ¿Quiénes son los responsables de los desaciertos deportivos que lo llevaron al descenso?". El Comercio, 06/12/2020.

Castillo, Raúl. "Yo también soy del Atalanta". El Comercio, 15/07/2020.

Chang, Jason \& Joshua Zenilman. "A Study of Sabermetrics in Major League Baseball: The Impact of Moneyball on Free Agent Salaries". Washington University in St. Louis, 2013.

Claeskens, Gerda \& Nils L. Hort. "Model Selection and Model Averaging". Cambridge, 2008.

Combe, Eduardo. "Resumen de la temporada 2020: todo lo que le pasó a Alianza Lima para descender a la Liga 2". El Comercio, 29/11/2020.

Corbeto, José A. “¿Cuáles son las posiciones en un equipo de fútbol?”. MyProtein, 2017.

Cruz, Christian [1]. "Alianza Lima: El corazón ha dejado de latir hasta que vuelvas (hasta que volvamos)". El Comercio, 29/11/2020.

Cruz, Christian [2]. "Alianza en el 2020: el fracaso de un equipo que ni siquiera llegó al promedio de un gol por encuentro". El Comercio,

El Universo. "Maldición a los que salen en la portada del FIFA, ahora el turno fue para Mbappé". 26/07/2020.

Félix Díaz, José. "La FIFA pide negociar una rebaja en los sueldos de los jugadores por el coronavirus". Marca, 26/03/2020.

FilmAffinity, "Moneyball: Rompiendo las reglas".

FootballCritic. "Top 50 Best Vetaran Players Older Than 32 Years”.

Hill, R.Carter; William E. Griffiths, y Guay C. Lim. "Principles of Econometrics ( $3^{\text {rd }}$ Ed.)". USA: John Wiley \& Sons, Inc., 2008.

IMDb. "El juego de la fortuna (2011)".

Lewis, Michael M. "Moneyball: The Art of Winning an Unfair Game (1st ed. reprinted)”. USA: W. W. Norton \& Co., 2004.

López, Luis. "La malidición de aparecer en una portada de FIFA". Hobbyconsolas, 28/09/2018.

Maupomé, Ana Paulina. “Moneyball, Liverpool's reason behing Jurgen Klopp’s hiring". As, 03/06/2019. 
McLeod, Josh, "The Engish Premier League is experiencing a textbook case of hyperinflation". The Conversation, 11/08/2017.

Peltz, Jim. "Sports salary inflation: \$1-million man march". Los Angeles Times, $18 / 12 / 2012$.

Pérez, Juan. "FIFA 21 ficha a Mbappé para una triple portada de lujo". El Desmarque eSports, 22/07/2020.

Redacción Gestión. "TAS declaró procedente pedido de Alianza Lima y deberá jugar en la Liga 1". Gestión, 17/03/21.

Rosales, Raúl. "Leicester City, la grandeza de un chico". El Comercio, 11/04/2016.

Stephenson, Debbie. "When Did Athletes Start Getting Rich?". Firmex, 24/06/2014.

TransferMarkt [1]. “\#20 Diogo Jota”. Accedido el 06/01/2021.

TransferMarkt [2]. “\#10 Allan Saint-Maximin”. Accedido el 07/01/2021.

Triola, Mario. "Elementary Statistics (9th Ed.)". USA: Pearson Addison Wesley, 2004.

\section{Notas al final}

1 Este ensayo es uno de investigación científica. El documento presenta los resultados originales de un proyecto terminado de investigación independiente. El tema de la investigación es Economía y Econometría.

2 Economista y Matemático. San Jose State University, California, EEUU. Magíster en Economía Aplicada, SJSU. Profesor Auxiliar a tiempo completo e investigador en la Facultad de Ciencias Económicas de la Universidad Nacional Mayor de San Marcos. Calle Germán Amezaga 375, Lima, Perú. E-mail: gmoyav@unmsm.edu.pe.

3 Bachiller en Economía. Universidad Nacional Mayor de San Marcos. Calle Germán Amezaga 375, Lima, Perú. E-mail: luis.cordova8@unmsm.edu.pe

4 El club descendió de categoría a fines del 2020, sin embargo, logró permanecer en la primera división del fútbol peruano debido a un fallo del Tribunal de Arbitraje Deportivo (TAS) emitido posteriormente a la redacción de este ensayo. (Gestión) 